\title{
THE CONTINUUM LIMIT AND QM-CONTINUUM APPROXIMATION OF QUANTUM MECHANICAL MODELS OF SOLIDS*
}

\author{
WEINAN $E^{\dagger}$ AND JIANFENG LU
}

\begin{abstract}
We consider the continuum limit for models of solids that arise in density functional theory and the QM-continuum approximation of such models. Two different versions of QMcontinuum approximation are proposed, depending on the level at which the Cauchy-Born rule is used, one at the level of electron density and one at the level of energy. Consistency at the interface between the smooth and the non-smooth regions is analyzed. We show that if the Cauchy-Born rule is used at the level of electron density, then the resulting QM-continuum model is free of the so-called "ghost force" at the interface. We also present dynamic models that bridge naturally the Car-Parrinello method and the QM-continuum approximation.
\end{abstract}

Key words. continuum limit, QM-continuum approximation, density functional theory

AMS subject classifications. 35Q40, 74Q05, 34E05, 74B20

\section{Introduction}

The present paper discusses two related topics: The continuum limit of the quantum mechanical models of solids and the QM-continuum approximation of such models. We will discuss models that arise from density functional theory (DFT), namely the Kohn-Sham DFT models and the Thomas-Fermi-von Weiszacker model [20]. Our derivation of the continuum limit in this paper is formal, and we will leave rigorous treatment to future papers. However, we think this formal treatment is important since it reveals some crucial insights of this limiting process. For example, it gives a procedure for finding high order approximations to the solutions of these models in the continuum limit. These approximations are useful in other contexts such as numerical computations. Indeed, our work here is partly motivated by the desire for developing efficient numerical algorithms for these models.

Our other main objective is to formulate coupled continuum-quantum mechanical models that use continuum models in part of the domain and the original quantum mechanical models in the rest of the domain. Such an approach is analogous in spirit to the quasi-continuum approximation or coupled atomistic-continuum methods $[2,9,22]$. Our main interest is to see whether additional errors arise at the interface between the continuum and QM regions. More specifically, we will study the size of the "ghost force", the force exerted on the atoms when they are in the equilibrium configuration, as a result of the coupling. In particular, we will formulate two different levels of QM-continuum approximation, one at the level of the electron density only, and the other at the level of both the electron density and the energy. We will show that there are no "ghost forces" in the first formulation.

These models form the basis on which we will develop efficient numerical algorithms that couple continuum and quantum models, as part of a joint project with Carlos García-Cervera and Jingfang Huang.

\footnotetext{
*Received: June 1, 2007; accepted (in revised version): June 20, 2007. Communicated by Shi Jin.

${ }^{\dagger}$ Department of Mathematics and PACM, Princeton University, Princeton, NJ 08544, USA (weinan@Princeton.edu).

${ }^{\ddagger}$ Program in Applied and Computational Mathematics, Princeton University, Princeton, NJ 08544, USA (jianfeng@math.Princeton.edu).
} 
The thermodynamic limit of the Thomas-Fermi type of model was considered before in [17], as well as [4]. The continuum limit of the Thomas-Fermi type of models was considered in [1]. The continuum limit of tight-binding models was considered in [10]. The quasi-continuum approximation of solids in the classical setting was proposed in [22], and has been extended by many people since then. Coupled continuum-atomistic approaches for the modeling of solids have also been discussed by many authors $[2,9]$. For a review, see [8]. The problem of "ghost force" for the quasi-continuum approximation in the classical setting was studied in [12, 21]. Great effort has gone into developing numerical algorithms for Kohn-Sham density functional theory. For a review, see [18]. Accurate numerical methods for orbital-free density functional theory were developed in [14]. Alternative methods for the Thomas-Fermivon Weiszacker model were presented in [16]. Attempts to construct algorithms in the spirit of the quasi-continuum approximation have been made in $[15,19]$. Related algorithms that combine quantum mechanical and classical models were presented in $[7]$.

\section{Setup for the continuum limit of atomistic models}

Given a fixed domain $\Omega$ in $\mathbb{R}^{3}$ and a fixed lattice $L$ with unit cell $\Gamma$, we consider a system of crystalline solids whose atoms are located at $x_{i} \in \varepsilon L \cap \Omega, i=1, \ldots, N$ in equilibrium. Here $\varepsilon$ is the lattice constant. Hence, the unit cells in the reference state are chosen to be $\varepsilon \Gamma+x_{i}$. It is easy to see that the total number of atoms in the system is $N=|\varepsilon L \cap \Omega|$, where $|\cdot|$ denotes the cardinal number of a set. We assume that each atom in the system has $n$ valence electrons. Therefore, the total number of valence electrons in the system is $n|\varepsilon L \cap \Omega|$.

In continuum theory, the deformation of a solid is described as a displacement field $u: \Omega \rightarrow R^{3}$ defined on the reference (undeformed) configuration. The position of a material point or an atom after deformation is located at $y=y(x)=(I+u)(x)$ where $x$ is the position in the reference configuration. $x$ is normally called the Lagrangian coordinate and $y$ is called the Eulerian coordinate. One of the problems we face when considering the continuum limit of electronic structure models of solids is the conflict between these coordinates: Continuum theory of solids such as elasticity theory naturally uses Lagrangian coordinates and electronic structure theory naturally uses Eulerian coordinates. As a result, we will have to specify carefully the variable with respect to which we carry out differentiation. This inevitably complicates the notation a bit.

In elasticity theory, we model the deformation of solids through a stored energy functional of the type

$$
\int_{\Omega} W(\nabla u(x)) d x
$$

where $W$ is the stored energy density. A key question is to find the function $W$. We will discuss how to find $W$ using electronic structure models. For notational ease, we denote by $A(x)=\nabla u(x)$ the Jacobian of $u$ at $x$.

\section{Quantum mechanical models of solids}

Under the Born-Oppenheimer approximation, the potential energy of a system is given entirely by the positions of the atoms, since the electronic structure is determined by the positions of the atoms. In principle, one can solve the many-body Schrödinger equation to find the electronic structure of the system from first principles. In practice, this is quite unfeasible. Therefore various approximate models 
involving fewer degrees of freedom are proposed. Among these models, three classes are most popular: the tight-binding (TB) models, orbital-free density functional theory (OFDFT) and Kohn-Sham (orbital-based) density functional theory (KSDFT). In this paper, we will focus on the KSDFT model and the Thomas-Fermi-von Weiszacker (TFW) model which belongs to the OFDFT class. At zero temperature, these models are formulated as variational problems.

Given the positions of the atoms, the charge distribution contributed by the ions of the system is given by

$$
m(y)=\sum_{x_{i} \in \varepsilon L \cap \Omega} m_{\varepsilon}^{a}\left(y-y\left(x_{i}\right)\right) .
$$

Here $m_{\varepsilon}^{a}$ is the rescaled charge distribution of one ion $m_{\varepsilon}^{a}(y)=m_{0}^{a}(y / \varepsilon) / \varepsilon^{3}$, given either by a Dirac distribution or a smooth pseudo-potential [18].

In KSDFT models, the energy functional of the system is a sum of the contributions from the kinetic energy of the electrons, the exchange-correlation energy, and the Coulomb interaction:

$$
\begin{aligned}
I_{\varepsilon}\left(\left\{\psi_{k}\right\}\right)=\varepsilon^{2} \sum_{k} \int_{\mathbb{R}^{3}}\left|\nabla \psi_{k}(y)\right|^{2} \mathrm{~d} y & +\int_{\mathbb{R}^{3}} \epsilon_{\mathrm{xc}}(\rho) \rho(y) \mathrm{d} y \\
& +\frac{\varepsilon}{2} \iint_{\mathbb{R}^{3} \times \mathbb{R}^{3}} \frac{(\rho-m)(y)(\rho-m)\left(y^{\prime}\right)}{\left|y-y^{\prime}\right|} \mathrm{d} y \mathrm{~d} y^{\prime} .
\end{aligned}
$$

The energy per atom of the system is then determined by the variational problem

$$
E_{\varepsilon}=\frac{1}{|\varepsilon L \cap \Omega|} \inf _{\left\{\psi_{k}\right\}} I_{\varepsilon}\left(\left\{\psi_{k}\right\}\right)
$$

Here $\left\{\psi_{k}(y)\right\}$ is a collection of orthonormal wave functions

$$
\int_{\mathbb{R}^{3}} \psi_{j}^{*}(y) \psi_{k}(y) \mathrm{d} y=\delta_{j k}
$$

The total number of these wave functions is equal to the number of occupied states (half the number of valence electrons due to Pauli exclusion principle), i.e., $n|\varepsilon L \cap \Omega| / 2$ (for simplicity, assume $n$ is an even number). $\rho$ is the electron density corresponding to the wave functions given by

$$
\rho(y)=2 \sum_{k}\left|\psi_{k}(y)\right|^{2}
$$

Since $\left\{\psi_{k}\right\}$ are orthonormal wave functions with total number $n|\varepsilon L \cap \Omega| / 2$, we have

$$
\int_{\mathbb{R}^{3}} \rho(y) \mathrm{d} y=n|\varepsilon L \cap \Omega| .
$$

In (3.2), $\epsilon_{\mathrm{xc}}$ is the exchange-correlation potential for which we have adopted the local density approximation. We assume

$$
\epsilon_{\mathrm{xc}}(\rho)=\epsilon_{\mathrm{xc}, 0}\left(\frac{1}{\varepsilon^{3}} \rho\left(\frac{y}{\varepsilon}\right)\right)
$$

so that the exchange-correlation energy scales properly when the lattice constant $\varepsilon$ changes. 
The Thomas-Fermi-von Weiszacker (TFW) model can be regarded as a simplification of the KSDFT models. While the Coulomb interaction part is retained, the kinetic part is approximated by functionals that depend only on the density (but not the orbitals):

$$
\varepsilon^{2} \int_{\mathbb{R}^{3}} \rho(y)^{5 / 3} \mathrm{~d} y+\varepsilon^{2} \int_{\mathbb{R}^{3}}|\nabla \sqrt{\rho(y)}|^{2} \mathrm{~d} y .
$$

To simplify the notation, we take $\nu(y)=\sqrt{\rho(y)}$ as the variable for the TFW model. Therefore the energy functional becomes

$$
\begin{aligned}
I_{\varepsilon}(\nu)=\varepsilon^{2} \int_{\mathbb{R}^{3}} \nu^{10 / 3} \mathrm{~d} y & +\varepsilon^{2} \int_{\mathbb{R}^{3}}|\nabla \nu|^{2} \mathrm{~d} y \\
& +\frac{\varepsilon}{2} \iint_{\mathbb{R}^{3} \times \mathbb{R}^{3}} \frac{\left(\nu^{2}-m\right)(y)\left(\nu^{2}-m\right)\left(y^{\prime}\right)}{\left|y-y^{\prime}\right|} \mathrm{d} y \mathrm{~d} y^{\prime} .
\end{aligned}
$$

The TFW functional is minimized under the normalization constraint:

$$
\int_{\mathbb{R}^{3}} \nu(y)^{2} \mathrm{~d} y=n|\varepsilon L \cap \Omega| .
$$

In order to prepare for the study of the continuum limit of these models, we have expressed them in a form that is properly scaled with $\varepsilon$.

\section{Asymptotic analysis of the continuum limit}

4.1. Thomas-Fermi-von Weiszacker model. We start the analysis with the TFW model. Consider the rescaled TFW energy functional:

$$
\begin{aligned}
I_{\varepsilon}(\nu)=\varepsilon^{2} \int_{\mathbb{R}^{3}} \nu^{10 / 3} \mathrm{~d} y & +\varepsilon^{2} \int_{\mathbb{R}^{3}}|\nabla \nu|^{2} \mathrm{~d} y \\
+ & \frac{\varepsilon}{2} \iint_{\mathbb{R}^{3} \times \mathbb{R}^{3}} \frac{\left(\nu^{2}-m\right)(y)\left(\nu^{2}-m\right)\left(y^{\prime}\right)}{\left|y-y^{\prime}\right|} \mathrm{d} y \mathrm{~d} y^{\prime} .
\end{aligned}
$$

The associated Euler-Lagrange equations are

$$
\begin{aligned}
-\varepsilon^{2} \Delta \nu+\frac{5}{3} \varepsilon^{2} \nu^{7 / 3}-\phi \nu+\lambda \nu & =0 \\
-\Delta \phi & =4 \pi \varepsilon\left(m-\nu^{2}\right),
\end{aligned}
$$

where $\lambda$ is a Lagrange multiplier for the normalization constraint and $\phi$ is the Coulombic potential generated by the electrons and the ions.

There are two scales in this problem: The scale of the displacement field, which is $\mathcal{O}(1)$, and the scale of the electron density, which is $\mathcal{O}(\varepsilon)$. To represent these two scales, we take the ansatz

$$
\begin{aligned}
& \nu=\nu\left(y, \frac{x}{\varepsilon}\right)=\frac{1}{\varepsilon^{3 / 2}} \nu_{0}\left(y, \frac{x}{\varepsilon}\right)+\frac{1}{\varepsilon^{1 / 2}} \nu_{1}\left(y, \frac{x}{\varepsilon}\right)+\varepsilon^{1 / 2} \nu_{2}\left(y, \frac{x}{\varepsilon}\right)+\cdots \\
& \phi=\phi\left(y, \frac{x}{\varepsilon}\right)=\phi_{0}\left(y, \frac{x}{\varepsilon}\right)+\varepsilon \phi_{1}\left(y, \frac{x}{\varepsilon}\right)+\varepsilon^{2} \phi_{2}\left(y, \frac{x}{\varepsilon}\right)+\cdots
\end{aligned}
$$

and also

$$
\lambda=\lambda_{0}+\varepsilon \lambda_{1}+\varepsilon^{2} \lambda_{2}+\cdots,
$$


where $x=x(y)=(I+u)^{-1}(y)$ is the Euler-Lagrange map: It gives the Lagrangian coordinate corresponding to $y . \nu(y, z)$ and $\phi(y, z)$ are functions defined on $\mathbb{R}^{3} \times \Gamma$ and periodic in the second variable on $\Gamma$. Note that we use the Eulerian coordinate for the first variable and the Lagrangian coordinate for the second variable. This is because we will impose periodicity in the second variable with a fixed period which is going to be the unit cell $\Gamma$ in the equilibrium configuration. Similarly, we have:

$$
m=m\left(y, \frac{x}{\varepsilon}\right)=\frac{1}{\varepsilon^{3}} m_{0}\left(y, \frac{x}{\varepsilon}\right)+\frac{1}{\varepsilon^{2}} m_{1}\left(y, \frac{x}{\varepsilon}\right)+\frac{1}{\varepsilon} m_{2}\left(y, \frac{x}{\varepsilon}\right)+\cdots .
$$

For example, we calculate

$$
m_{0}(y, z)=\sum_{z_{i} \in L} m_{0}^{a}\left((I+A)(x)\left(z-z_{i}\right)\right)
$$

where $A=\nabla u$ is evaluated at $x=x(y)$.

We now have

$$
-\Delta f\left(y, \frac{x}{\varepsilon}\right)=-\Delta_{1} f\left(y, \frac{x}{\varepsilon}\right)-\frac{1}{\varepsilon} \nabla_{2}^{x} \cdot \nabla_{1} f\left(y, \frac{x}{\varepsilon}\right)-\frac{1}{\varepsilon} \nabla_{1} \cdot \nabla_{2}^{x} f\left(y, \frac{x}{\varepsilon}\right)-\frac{1}{\varepsilon^{2}} \Delta_{2}^{x} f\left(y, \frac{x}{\varepsilon}\right) .
$$

Here operators like $\nabla_{1}$ take the derivative with respect to the first variable and $\nabla_{2}$ the second. We define $\nabla_{2}^{x}=(I+\nabla u(x))^{-\mathrm{T}} \nabla_{2}$ and $\Delta_{2}^{x}=\nabla_{2}^{x} \cdot \nabla_{2}^{x}$ to simplify the notation. The factors $(I+\nabla u(x))^{-\mathrm{T}}$ arise as a result of transformation between the Eulerian and Lagrangian coordinates.

Expanding the Euler-Lagrange equations, we get the leading order equations:

$$
\begin{aligned}
-\Delta_{2}^{x} \nu_{0}+\frac{5}{3} \nu_{0}^{7 / 3}-\phi_{0} \nu_{0}+\lambda_{0} \nu_{0} & =0 ; \\
-\Delta_{2}^{x} \phi_{0} & =4 \pi\left(m_{0}-\nu_{0}^{2}\right) .
\end{aligned}
$$

The next order equations are

$$
\begin{aligned}
-\Delta_{2}^{x} \nu_{1}- & \nabla_{1} \cdot \nabla_{2}^{x} \nu_{0}-\nabla_{2}^{x} \cdot \nabla_{1} \nu_{0} \\
& +\frac{35}{9} \nu_{0}^{4 / 3} \nu_{1}-\phi_{1} \nu_{0}-\phi_{0} \nu_{1}+\lambda_{1} \nu_{0}+\lambda_{0} \nu_{1}=0 \\
-\Delta_{2}^{x} \phi_{1} & -\nabla_{1} \cdot \nabla_{2}^{x} \phi_{0}-\nabla_{2}^{x} \cdot \nabla_{1} \phi_{0}=4 \pi\left(m_{1}-2 \nu_{0} \nu_{1}\right) .
\end{aligned}
$$

The leading order equations (4.9)-(4.10) are a set of equations for functions defined on the unit cell $\Gamma$, with $A=\nabla u(x)$ as parameters.

Define the periodic Thomas-Fermi-von Weiszacker functional

$$
\begin{aligned}
& I_{A}(\nu)=\int_{\Gamma} \nu^{10 / 3}(z ; A) \mathrm{d} z+\int_{\Gamma}\left|(I+A)^{-\mathrm{T}} \nabla \nu(z ; A)\right|^{2} \mathrm{~d} z \\
& \quad+\frac{1}{2} \iint_{\Gamma \times \Gamma}\left(\nu^{2}-m_{\mathrm{CB}}\right)(z ; A) G\left(z-z^{\prime} ; A\right)\left(\nu^{2}-m_{\mathrm{CB}}\right)\left(z^{\prime} ; A\right) \mathrm{d} z \mathrm{~d} z^{\prime},
\end{aligned}
$$

where $m_{\mathrm{CB}}(z ; A(x))=m_{0}(y, z)=\sum_{z_{i}} m_{0}^{a}\left((I+A)(x)\left(z-z_{i}\right)\right)$ and $G(z ; A)$ is the periodic Coulombic kernel [17] which is the solution of

$$
-\left((I+A)^{-\mathrm{T}} \nabla\right) \cdot\left((I+A)^{-\mathrm{T}} \nabla\right) G(z ; A)=4 \pi\left(\delta(z)-\frac{1}{|\Gamma|}\right) \text { in } \Gamma
$$


with periodic boundary condition and $\lim _{z \rightarrow 0}(G(z ; A)-1 /|(I+A) z|)=0$.

It is easy to see that the Euler-Lagrange equation of $I_{A}(\nu)$ are the same as (4.9)(4.10) with $A=\nabla u(x)$. The second order equations (4.11)-(4.12) give the next order approximation which we omit here. Define the stored energy density $W(A, \nu)$ as

$$
W(A, \nu)=\frac{\operatorname{det}(I+A)}{|\Gamma|} I_{A}(\nu)
$$

Therefore the minimizer $\nu_{\mathrm{CB}}(z ; A)$ of the variational problem

$$
W_{\mathrm{CB}}(A)=\inf _{\nu} W(A, \nu(\cdot ; A))
$$

with normalization constraint

$$
\int_{\Gamma} \nu^{2}(z ; A) \mathrm{d} z=\frac{n}{\operatorname{det}(I+A)} .
$$

gives the leading order approximation of $\nu(y, z)=\nu_{\mathrm{CB}}(z ; \nabla u(x))$, where $y=(I+u)(x)$. We call $\nu_{\mathrm{CB}}$ the Cauchy-Born (square root of) electron density and $W_{\mathrm{CB}}$ the CauchyBorn stored energy density.

We conclude that the continuum limit of energy in the TFW model is given by

$$
E=\int_{\Omega} W_{\mathrm{CB}}(\nabla u(x)) \mathrm{d} x .
$$

Rigorous analysis for the TFW model can be found in $[1,4,17]$.

4.2. Kohn-Sham density functional theory. Next, we consider the continuum limit for models from Kohn-Sham density functional theory. As discussed in Section 3, the major difference between the KSDFT models and the TFW model is in the kinetic energy part as well as the exchange-correlation energy. As we will see below, the analysis of the KSDFT models, while similar to the TFW model, contains some new insights.

The rescaled energy functional for the Kohn-Sham density functional theory model is

$$
\begin{aligned}
I_{\varepsilon}\left(\left\{\psi_{k}\right\}\right)=\varepsilon^{2} \sum_{k} \int_{\mathbb{R}^{3}}\left|\nabla \psi_{k}(y)\right|^{2} \mathrm{~d} y & +\int_{\mathbb{R}^{3}} \epsilon_{\mathrm{xc}}(\rho) \rho(y) \mathrm{d} y \\
& +\frac{\varepsilon}{2} \iint_{\mathbb{R}^{3} \times \mathbb{R}^{3}} \frac{(\rho-m)(y)(\rho-m)\left(y^{\prime}\right)}{\left|y-y^{\prime}\right|} \mathrm{d} y \mathrm{~d} y^{\prime} .
\end{aligned}
$$

The associated Euler-Lagrange equations are

$$
\begin{aligned}
-\varepsilon^{2} \Delta \psi_{k}+V_{\mathrm{xc}}(\rho) \psi_{k}-\phi \psi_{k}+\sum_{k^{\prime}} \lambda_{k k^{\prime}} \psi_{k^{\prime}} & =0 \\
-\Delta \phi & =4 \pi \varepsilon(m-\rho) .
\end{aligned}
$$

Here the $\lambda$ 's are the Lagrange multipliers for the orthonormality constraints, $\phi$ is the Coulombic potential generated by the electrons and the ions, and $V_{\mathrm{xc}}(\rho)=\epsilon_{\mathrm{xc}}(\rho)+$ $\epsilon_{\mathrm{xc}}^{\prime}(\rho) \rho$.

The orbitals $\left\{\psi_{k}\right\}$ are far from unique. We will assume that the collection $\left\{\psi_{k}\right\}$ can be chosen as $\left\{\psi_{\alpha}\left(y_{i},\left(x-x_{i}\right) / \varepsilon\right)\right\}$, where $\alpha$ ranging from 1 to $n / 2$ is the index for 
the occupied states. $\psi_{\alpha}(y, \cdot)$ is localized at 0 , i.e., it decays away from the origin. Thus $\psi_{\alpha}\left(y_{i},\left(x-x_{i}\right) / \varepsilon\right)$ is localized at the atom position $x_{i}$. If the system is in equilibrium or under homogeneous deformation, $\left\{\psi_{\alpha}\left(y_{i},\left(x-x_{i}\right) / \varepsilon\right)\right\}$ can be chosen as the well-known Wannier function [23] of the $\alpha$-th energy band centered at $x_{i}$.

Similar to the case of the TFW model, we take the following ansatz:

$$
\begin{aligned}
\psi_{\alpha}(y, z) & =\frac{1}{\varepsilon^{3 / 2}} \psi_{\alpha, 0}(y, z)+\frac{1}{\varepsilon^{1 / 2}} \psi_{\alpha, 1}(y, z)+\varepsilon^{1 / 2} \psi_{\alpha, 2}(y, z)+\cdots, \\
\rho & =\rho\left(y, \frac{x}{\varepsilon}\right)=\frac{1}{\varepsilon^{3}} \rho_{0}\left(y, \frac{x}{\varepsilon}\right)+\frac{1}{\varepsilon^{2}} \rho_{1}\left(y, \frac{x}{\varepsilon}\right)+\frac{1}{\varepsilon^{1}} \rho_{2}\left(y, \frac{x}{\varepsilon}\right)+\cdots, \\
\phi & =\phi\left(y, \frac{x}{\varepsilon}\right)=\phi_{0}\left(y, \frac{x}{\varepsilon}\right)+\varepsilon \phi_{1}\left(y, \frac{x}{\varepsilon}\right)+\varepsilon^{2} \phi_{2}\left(y, \frac{x}{\varepsilon}\right)+\cdots
\end{aligned}
$$

As discussed above, $\psi_{\alpha}(y, z)$ decays when $z$ becomes large, and $\rho(y, z)$ and $\phi(y, z)$ are periodic in $z$ as in the TFW model. Recall that

$$
\rho(y)=2 \sum_{\alpha} \sum_{x_{j} \in \varepsilon L \cap \Omega}\left|\psi_{\alpha}\left(y_{j}, \frac{x-x_{j}}{\varepsilon}\right)\right|^{2} .
$$

In the limit as $\varepsilon \rightarrow 0$, by the decay property, we obtain at leading order

$$
\rho_{0}(y, z)=2 \sum_{\alpha} \sum_{z_{j} \in L}\left|\psi_{\alpha, 0}\left(y, z-z_{j}\right)\right|^{2} .
$$

Note that $\psi_{\alpha, 0}\left(y, \cdot-z_{j}\right)$ is a translation of $\psi_{\alpha, 0}\left(y, \cdot-z_{k}\right)$ with translation vector $z_{j}-z_{k}$. Similarly, for the orthonormality constraint, we have in the limit

$$
\int_{\mathbb{R}^{3}} \psi_{\alpha, 0}^{*}\left(y, z-z_{i}\right) \psi_{\alpha^{\prime}, 0}\left(y, z-z_{j}\right) \mathrm{d} z=\delta_{\alpha \alpha^{\prime}} \delta_{i j} / \operatorname{det}((I+\nabla u)(x)) .
$$

Taking these into consideration and expanding the Euler-Lagrange equations, we get the leading order equations:

$$
\begin{aligned}
-\Delta_{2}^{x} \psi_{\alpha, 0}(y, z) & +V_{x c, 0}\left(\rho_{0}\right) \psi_{\alpha, 0}(y, z)-\phi_{0}(y, z) \psi_{\alpha, 0}(y, z) \\
& +\sum_{\alpha^{\prime}, z_{j} \in L} \lambda_{\alpha \alpha^{\prime}, z_{j}} \psi_{\alpha^{\prime}, 0}\left(y, z-z_{j}\right)=0 \\
-\Delta_{2}^{x} \phi_{0}(y, z)= & 4 \pi\left(m_{0}-\rho_{0}\right)(y, z) .
\end{aligned}
$$

Here the $\lambda$ 's are Lagrange multipliers.

Define the functional

$$
\begin{aligned}
& I_{A}(\{\psi\})=\sum_{\alpha} \int_{\mathbb{R}^{3}}\left|(I+A)^{-\mathrm{T}} \nabla \psi_{\alpha}(z ; A)\right|^{2} \mathrm{~d} z+\int_{\Gamma} \epsilon_{\mathrm{xc}, 0}(\rho(z ; A)) \rho(z ; A) \mathrm{d} z \\
& \quad+\frac{1}{2} \iint_{\Gamma \times \Gamma}\left(\rho-m_{\mathrm{CB}}\right)(z ; A) G\left(z-z^{\prime} ; A\right)\left(\rho-m_{\mathrm{CB}}\right)\left(z^{\prime} ; A\right) \mathrm{d} z \mathrm{~d} z^{\prime},
\end{aligned}
$$

where $m_{\mathrm{CB}}(z ; A(x))=m_{0}(y, z)=\sum_{z_{i}} m_{0}^{a}\left((I+A)(x)\left(z-z_{i}\right)\right)$ and $G(z ; A)$ is the periodic Coulombic kernel as in (4.14). The stored energy density $W(A,\{\psi\})$ is given by

$$
W(A,\{\psi\})=\frac{\operatorname{det}(I+A)}{|\Gamma|} I_{A}(\{\psi\})
$$


Similar asymptotics as in the TFW model showsthat the minimizer $\psi_{\mathrm{CB}, \alpha}$ of the variational problem

$$
W_{\mathrm{CB}}(A)=\inf _{\{\psi\}} W\left(A,\left\{\psi_{\alpha}(\cdot ; A)\right\}\right)
$$

with orthonormality constraints

$$
\int_{\mathbb{R}^{3}} \psi_{\alpha, 0}^{*}\left(z-z_{i} ; A\right) \psi_{\alpha, 0}\left(z-z_{j} ; A\right) \mathrm{d} z=\delta_{\alpha \alpha^{\prime}} \delta_{i j} / \operatorname{det}(I+A) .
$$

gives the leading order approximation of $\psi_{\alpha}(y, z)$. We call $\psi_{\mathrm{CB}, \alpha}$ the Cauchy-Born wave functions and $W_{\mathrm{CB}}$ the Cauchy-Born stored energy density.

Note that for KSDFT, the cell problem is not formulated as a periodic problem over the unit cell, but rather a problem over the whole space, as shown in (4.29). The wave function, although localized, is also defined on the whole $\mathbb{R}^{3}$.

We further remark that, although (4.29) is derived from assumptions of periodicity, it takes a form that is quite different from the usual formulation of periodic models when wave functions are included (e.g., for the Hartree and Hartree-Fock model as in $[6,5])$. Our formulation $(4.29)$ is in real space, not in $k$-space where the Bloch boundary condition is used [18]. Because of this, we have chosen a localized representation (Wannier functions) for the electronic states since they are more naturally associated with each individual atom or electron. For numerical computation, the real space formulation might be more advantageous than the $k$-space representation. This point will be further discussed in future publications.

The asymptotic analysis reveals something that may seem surprising at a first sight, namely that the problem becomes effectively local: all terms in the asymptotic expansion are determined by solving differential equations in the fast variables; the slow variables enter only as parameters. In the language of fiber bundles [11], all terms are determined by solving PDEs on the fibers - differentiation with respect to the variables on the base manifold only enters in the source terms. Indeed given the displacement field, the leading order terms become independent for different values of the slow variable.

\section{Analysis of the QM-continuum approximation}

We next consider the QM-continuum approximation of the energy functional. We will formulate a QM-continuum model based on the philosophy of domain decomposition. The domain $\Omega$ is decomposed into two regions, the non-smooth region $\Omega_{n s}$ and the smooth region $\Omega_{s}$, according to the smoothness of the displacement field. In the smooth region, the deformation is locally close to being homogeneous; therefore, we can adopt the Cauchy-Born rule. The non-smooth region might contain defects. Thus the original model is kept.

The Cauchy-Born rule gives us information for both electron density and energy. Accordingly, we can imagine two different strategies for using the Cauchy-Born rule. The first is to use only the Cauchy-Born electron density. The second is to use also the Cauchy-Born energy. We call the former Model A and the latter Model B. Their main difference lies in the treatment of the long-range Coulomb interaction. As we have seen above, the Kohn-Sham DFT models treat the Coulombic interaction in the same way with the TFW model. Hence, for simplicity, we will provide the detailed analysis of the QM-continuum approximation only for the TFW model. For the KSDFT models, the same analysis applies with trivial modifications. We thus skip most of the details. 
5.1. QM-continuum approximation of the TFW model: Model A. According to the philosophy of domain decomposition, we write the electron density as a sum of the smooth and non-smooth terms:

$$
\nu(y)=\nu_{s}(y) 1_{y\left(\Omega_{s}\right)}+\nu_{n s}(y) 1_{y\left(\Omega_{n s}\right)} .
$$

In the smooth region, we approximate the electron density by the Cauchy-Born rule:

$$
\nu_{s}(y)=\nu_{\mathrm{CB}}\left(\frac{x}{\varepsilon} ; \nabla u(x)\right)
$$

where $x=x(y)$ is the Euler-Lagrange map. The non-smooth electron density $\nu_{n s}$ satisfies the boundary condition

$$
\nu_{n s}(y)=\nu_{s}(y) \quad \text { on } \partial y\left(\Omega_{n s}\right),
$$

in order to guarantee that the electron density is continuous at the interface.

Substituting (5.1) into the energy functional (4.1), we get

$$
\begin{aligned}
I_{\varepsilon}^{A}\left(\nu_{n s}\right)=I_{n s}\left(\nu_{n s}\right) & +\varepsilon^{2} \int_{y\left(\Omega_{s}\right)} \nu_{s}^{10 / 3} \mathrm{~d} y+\varepsilon^{2} \int_{y\left(\Omega_{s}\right)}\left|\nabla \nu_{s}\right|^{2} \mathrm{~d} y \\
& +\frac{\varepsilon}{2} \iint_{y\left(\Omega_{s}\right) \times y\left(\Omega_{s}\right)} \frac{\left(\nu_{s}^{2}-m\right)(y)\left(\nu_{s}^{2}-m\right)\left(y^{\prime}\right)}{\left|y-y^{\prime}\right|} \mathrm{d} y \mathrm{~d} y^{\prime} .
\end{aligned}
$$

Here $I_{n s}\left(\nu_{n s}\right)$ is given by

$$
\begin{aligned}
I_{n s}\left(\nu_{n s}\right)=\varepsilon^{2} \int_{y\left(\Omega_{n s}\right)} \nu_{n s}^{10 / 3} \mathrm{~d} y & +\varepsilon^{2} \int_{y\left(\Omega_{n s}\right)}\left|\nabla \nu_{n s}\right|^{2} \mathrm{~d} y \\
& +\frac{\varepsilon}{2} \iint_{y\left(\Omega_{n s}\right) \times y\left(\Omega_{n s}\right)} \frac{\left(\nu_{n s}^{2}-m\right)(y)\left(\nu_{n s}^{2}-m\right)\left(y^{\prime}\right)}{\left|y-y^{\prime}\right|} \mathrm{d} y \mathrm{~d} y^{\prime} \\
& +\varepsilon \iint_{y\left(\Omega_{s}\right) \times y\left(\Omega_{n s}\right)} \frac{\left(\nu_{s}^{2}-m\right)(y)\left(\nu_{n s}^{2}-m\right)\left(y^{\prime}\right)}{\left|y-y^{\prime}\right|} \mathrm{d} y \mathrm{~d} y^{\prime} .
\end{aligned}
$$

The energy of the system is given by

$$
E_{\varepsilon}^{A}=\inf _{\nu_{n s}} I_{\varepsilon}^{A}\left(\nu_{n s}\right) .
$$

The associated Euler-Lagrange equations of $I_{n s}$ are

$$
\begin{aligned}
-\varepsilon^{2} \Delta \nu_{n s}+\frac{5}{3} \varepsilon^{2} \nu_{n s}^{7 / 3}-\left(\phi_{n s}+\phi_{s}\right) \nu_{n s}+\lambda \nu_{n s} & =0, \\
-\Delta \phi_{n s} & =4 \pi \varepsilon\left(m-\nu_{n s}^{2}\right) 1_{y\left(\Omega_{n s}\right)}
\end{aligned}
$$

where $\phi_{s}$ is the Coulombic potential generated by the charges in the smooth region (taking the Cauchy-Born approximation).

We now examine the accuracy of this QM-continuum model. For this purpose, we consider the situation when the crystal is under homogeneous deformation. In this case, the Cauchy-Born rule is exact. We assume that $\Omega=\mathbb{R}^{3}$ and $y(x)=(I+A) x$ with $A$ fixed. In this case, the minimizer for the whole system is $\nu^{0}(y)=\nu_{\mathrm{CB}}(x / \varepsilon ; A)$, from the definition of $\nu_{\mathrm{CB}}$. Note that if $\nu_{n s}$ is an arbitrary electron density function satisfying the boundary condition (5.3), $\nu=\nu_{s} 1_{y\left(\Omega_{s}\right)}+\nu_{n s} 1_{y\left(\Omega_{n s}\right)}$ is then a possible 
electron density function for the whole system. If we take $\nu_{n s}^{0}(y)=\nu_{\mathrm{CB}}(x / \varepsilon ; A)$ in $y\left(\Omega_{n s}\right)$, which certainly satisfies (5.3), we have

$$
I_{\varepsilon}^{A}\left(\nu_{n s}^{0}\right)=I_{\varepsilon}\left(\nu^{0}\right) \leq I_{\varepsilon}(\nu)=I_{\varepsilon}^{A}\left(\nu_{n s}\right) .
$$

Hence $\nu_{n s}^{0}$ is the minimizer of (5.6). This means that model A yields the correct minimizer in the homogeneous case.

To calculate the force, it is more convenient to use the Lagrangian coordinates. The energy functional (5.4) rewritten in Lagrangian coordinates is

$$
\begin{aligned}
I_{\varepsilon}^{A}= & \varepsilon^{2} \int_{\Omega_{s}} \varepsilon^{2} \nu_{s}^{10 / 3}(y) \operatorname{det}(I+A) \mathrm{d} x+\varepsilon^{2} \int_{\Omega_{s}}\left|\nabla \nu_{s}(y)\right|^{2} \operatorname{det}(I+A) \mathrm{d} x \\
& +\frac{\varepsilon}{2} \iint_{\Omega_{s} \times \Omega_{s}} \frac{\left(\nu_{s}^{2}-m\right)(y)\left(\nu_{s}^{2}-m\right)\left(y^{\prime}\right)}{\left|y-y^{\prime}\right|} \operatorname{det}(I+A) \operatorname{det}\left(I+A^{\prime}\right) \mathrm{d} x \mathrm{~d} x^{\prime} \\
& +\varepsilon^{2} \int_{\Omega_{n s}} \nu_{n s}^{10 / 3}(y) \operatorname{det}(I+A) \mathrm{d} x+\varepsilon^{2} \int_{\Omega_{n s}}\left|\nabla \nu_{n s}(y)\right|^{2} \operatorname{det}(I+A) \mathrm{d} x \\
& +\frac{\varepsilon}{2} \iint_{\Omega_{n s} \times \Omega_{n s}} \frac{\left(\nu_{n s}^{2}-m\right)(y)\left(\nu_{n s}^{2}-m\right)\left(y^{\prime}\right)}{\left|y-y^{\prime}\right|} \operatorname{det}(I+A) \operatorname{det}\left(I+A^{\prime}\right) \mathrm{d} x \mathrm{~d} x^{\prime} \\
& +\varepsilon \iint_{\Omega_{s} \times \Omega_{n s}} \frac{\left(\nu_{s}^{2}-m\right)(y)\left(\nu_{n s}^{2}-m\right)\left(y^{\prime}\right)}{\left|y-y^{\prime}\right|} \operatorname{det}(I+A) \operatorname{det}\left(I+A^{\prime}\right) \mathrm{d} x \mathrm{~d} x^{\prime},
\end{aligned}
$$

where for notational ease, we have used $y, y^{\prime}, A$, and $A^{\prime}$ as shorthand for $y(x), y\left(x^{\prime}\right)$, $A(x)$ and $A\left(x^{\prime}\right)$ respectively. The variational derivative (in the Gateaux sense) is:

$$
\begin{aligned}
& \frac{\delta}{\delta u} E_{\varepsilon}^{A}[h] \\
= & \frac{10}{3} \varepsilon^{2} \int_{\Omega_{s}} \nu_{s}^{7 / 3} \frac{\delta \nu_{s}}{\delta A_{i j}} \frac{\partial h_{i}}{\partial x_{j}} \operatorname{det}(I+A) \mathrm{d} x \\
& +2 \varepsilon^{2} \int_{\Omega_{s}} \frac{\delta \nabla \nu_{s}}{\delta A_{i j}} \frac{\partial h_{i}}{\partial x_{j}} \cdot \nabla \nu_{s} \operatorname{det}(I+A) \mathrm{d} x \\
& +\varepsilon \iint_{\Omega_{s} \times \Omega} \frac{\left(2 \nu_{s} \frac{\delta \nu_{s}}{\delta A_{i j}} \frac{\partial h_{i}}{\partial x_{j}}\right)(y)\left(\nu^{2}-m\right)\left(y^{\prime}\right)}{\left|y-y^{\prime}\right|} \operatorname{det}(I+A) \operatorname{det}\left(I+A^{\prime}\right) \mathrm{d} x \mathrm{~d} x^{\prime} \\
& -\varepsilon \iint_{\Omega \times \Omega} \frac{\left(\frac{\delta m}{\delta u}[h]\right)(y)\left(\nu^{2}-m\right)\left(y^{\prime}\right)}{\left|y-y^{\prime}\right|} \operatorname{det}(I+A) \operatorname{det}\left(I+A^{\prime}\right) \mathrm{d} x \mathrm{~d} x^{\prime} \\
& +\varepsilon^{2} \int_{\Omega} \nu^{10 / 3} \frac{\partial \operatorname{det}(I+A)}{\partial A_{i j}} \frac{\partial h_{i}}{\partial x_{j}} \mathrm{~d} x+\varepsilon^{2} \int_{\Omega}|\nabla \nu|^{2} \frac{\partial \operatorname{det}(I+A)}{\partial A_{i j}} \frac{\partial h_{i}}{\partial x_{j}} \mathrm{~d} x \\
& +\varepsilon \iint_{\Omega \times \Omega} \frac{\left(\nu^{2}-m\right)(y)\left(\nu^{2}-m\right)\left(y^{\prime}\right)}{\left|y-y^{\prime}\right|} \frac{\partial \operatorname{det}(I+A)}{\partial A_{i j}} \frac{\partial h_{i}}{\partial x_{j}} \operatorname{det}\left(I+A^{\prime}\right) \mathrm{d} x \mathrm{~d} x^{\prime} \\
& -\varepsilon \iint_{\Omega \times \Omega} \frac{\left(\nu^{2}-m\right)(y)\left(\nu^{2}-m\right)\left(y^{\prime}\right)}{\left|y-y^{\prime}\right|^{3}}\left(y-y^{\prime}\right) \cdot h(x) \operatorname{det}(I+A) \operatorname{det}\left(I+A^{\prime}\right) \mathrm{d} x \mathrm{~d} x^{\prime} .
\end{aligned}
$$

The first three terms on the right hand side are contributions from the dependence of $\nu_{\mathrm{CB}}$ on the deformation; the next one represents the dependence of the ion charge distribution on the deformation; the last four terms are from the coordinate change.

One of the most important issues for this type of multi-scale model is the existence of the "ghost force": When the system is in equilibrium, the force is not equal to zero due to the coupling. The existence of the "ghost force" characterizes the loss of 
accuracy at the interface between smooth and non-smooth regions [12]. To see whether "ghost force" exists for the QM-continuum model under discussion, let us consider the equilibrium state: $y(x)=x$ and take $\Omega=\mathbb{R}^{3}\left(\Omega_{s}=\mathbb{R}^{3} \backslash \Omega_{n s}\right)$ to avoid effects at the boundary of the whole system, since we care only about the behavior at the interface between smooth and non-smooth regions caused by the coupling. Under these assumptions, (5.9) becomes

$$
\begin{aligned}
\frac{\delta}{\delta u} E_{\varepsilon}^{A}[h]= & \frac{10}{3} \varepsilon^{2} \int_{\Omega_{s}} \nu_{s}^{7 / 3} \frac{\delta \nu_{s}}{\delta A_{i j}} \frac{\partial h_{i}}{\partial x_{j}} \mathrm{~d} x+2 \varepsilon^{2} \int_{\Omega_{s}} \frac{\delta \nabla \nu_{s}}{\delta A_{i j}} \frac{\partial h_{i}}{\partial x_{j}} \cdot \nabla \nu_{s} \mathrm{~d} x \\
& +\varepsilon \iint_{\Omega_{s} \times \Omega} \frac{\left(2 \nu_{s} \frac{\delta \nu_{s}}{\delta A_{i j}} \frac{\partial h_{i}}{\partial x_{j}}\right)(x)\left(\nu^{2}-m\right)\left(x^{\prime}\right)}{\left|x-x^{\prime}\right|} \mathrm{d} x \mathrm{~d} x^{\prime} \\
& -\varepsilon \iint_{\Omega \times \Omega} \frac{\left(\frac{\delta m}{\delta u}[h]\right)(x)\left(\nu^{2}-m\right)\left(x^{\prime}\right)}{\left|x-x^{\prime}\right|} \mathrm{d} x \mathrm{~d} x^{\prime} \\
& +\int \varepsilon_{\Omega}^{2} \nu^{10 / 3} \frac{\partial \operatorname{det}(I+A)}{\partial A_{i j}} \frac{\partial h_{i}}{\partial x_{j}} \mathrm{~d} x+\varepsilon^{2} \int_{\Omega}|\nabla \nu|^{2} \frac{\partial \operatorname{det}(I+A)}{\partial A_{i j}} \frac{\partial h_{i}}{\partial x_{j}} \mathrm{~d} x \\
& +\varepsilon \iint_{\Omega \times \Omega} \frac{\left(\nu^{2}-m\right)(x)\left(\nu^{2}-m\right)\left(x^{\prime}\right)}{\left|x-x^{\prime}\right|} \frac{\partial \operatorname{det}(I+A)}{\partial A_{i j}} \frac{\partial h_{i}}{\partial x_{j}} \mathrm{~d} x \mathrm{~d} x^{\prime} \\
& -\varepsilon \iint_{\Omega \times \Omega} \frac{\left(\nu^{2}-m\right)(x)\left(\nu^{2}-m\right)\left(x^{\prime}\right)}{\left|x-x^{\prime}\right|^{3}}\left(x-x^{\prime}\right) \cdot h(x) \mathrm{d} x \mathrm{~d} x^{\prime} .
\end{aligned}
$$

Here $\partial \operatorname{det}(I+A) / \partial A_{i j}$ is evaluated at $A=0$; hence it can be taken out of the integral. Moreover, $\nu$ and $m$ are periodic with respect to the unit cell $\Gamma$, since the system is in equilibrium.

Without loss of generality, we assume that $h$ corresponds to a trial displacement of the atom located at 0 , i.e., $h$ is supported in the cube $[-\varepsilon, \varepsilon]^{3}$, tri-linear (linear in each dimension) and symmetric with respect to each coordinate plane. The sum of the first three terms in (5.10),

$$
\begin{array}{rl}
\frac{10}{3} \varepsilon^{2} \int_{\Omega_{s}} \nu_{s}^{7 / 3} \frac{\delta \nu_{s}}{\delta A_{i j}} \frac{\partial h_{i}}{\partial x_{j}} \mathrm{~d} & x+2 \varepsilon^{2} \int_{\Omega_{s}} \frac{\delta \nabla \nu_{s}}{\delta A_{i j}} \frac{\partial h_{i}}{\partial x_{j}} \cdot \nabla \nu_{s} \mathrm{~d} x \\
+\varepsilon \iint_{\Omega_{s} \times \Omega} \frac{\left(2 \nu_{s} \frac{\delta \nu_{s}}{\delta A_{i j}} \frac{\partial h_{i}}{\partial x_{j}}\right)(x)\left(\nu^{2}-m\right)\left(x^{\prime}\right)}{\left|x-x^{\prime}\right|} \mathrm{d} x \mathrm{~d} x^{\prime}
\end{array}
$$

vanishes since $\nu_{\mathrm{CB}}$ is the minimizer of the periodic problem. The remaining terms vanish due to symmetry. For example, let us consider the term

$$
\int_{\Omega} \nu^{10 / 3}(x) \frac{\partial h_{i}}{\partial x_{j}}(x) \mathrm{d} x .
$$

Due to symmetry, we have

$$
\begin{aligned}
& \frac{\partial h_{i}}{\partial x_{j}}\left(x^{1}, x^{2}, x^{3}\right)=-\frac{\partial h_{i}}{\partial x_{j}}\left(-x^{1}, x^{2}, x^{3}\right), \\
& \nu^{10 / 3}\left(x^{1}, x^{2}, x^{3}\right)=\nu^{10 / 3}\left(-x^{1}, x^{2}, x^{3}\right) .
\end{aligned}
$$

It is easy to see that the integral (5.11) is equal to zero. It follows from a similar analysis that the other remaining terms also vanish.

We conclude that for model A, no "ghost force" appears. Hence, model A is first order uniform accurate as discussed in [12]. This is natural since we include explicitly the long range interaction in the approximated energy functional (5.6). 
5.2. QM-continuum approximation of the TFW model: Model B. For model B, we further simplify (5.6) by using the Cauchy-Born energy for the smooth region:

$$
I_{\varepsilon}^{B}\left(\nu_{n s}\right)=I_{n s}\left(\nu_{n s}\right)+\int_{\Omega_{s}} W_{\mathrm{CB}}(\nabla u(x)) \mathrm{d} x
$$

with $I_{n s}$ given by

$$
\begin{aligned}
I_{n s}\left(\nu_{n s}\right)= & \inf _{\nu_{n s}} \varepsilon^{2} \int_{y\left(\Omega_{n s}\right)} \nu_{n s}^{10 / 3} \mathrm{~d} y+\varepsilon^{2} \int_{y\left(\Omega_{n s}\right)}\left|\nabla \nu_{n s}\right|^{2} \mathrm{~d} y \\
& +\frac{\varepsilon}{2} \iint_{y\left(\Omega_{n s}\right) \times y\left(\Omega_{n s}\right)} \frac{\left(\nu_{n s}^{2}-m\right)(y)\left(\nu_{n s}^{2}-m\right)\left(y^{\prime}\right)}{\left|y-y^{\prime}\right|} \mathrm{d} y \mathrm{~d} y^{\prime} \\
& +\varepsilon \iint_{y\left(\Omega_{n s}\right) \times y\left(\Omega_{s}\right)} \frac{\left(\nu_{n s}^{2}-m\right)(y)\left(\nu_{s}^{2}-m\right)\left(y^{\prime}\right)}{\left|y-y^{\prime}\right|} \mathrm{d} y \mathrm{~d} y^{\prime} .
\end{aligned}
$$

The energy of the system is given by

$$
E_{\varepsilon}^{B}=\inf _{\nu_{n s}} I_{\varepsilon}^{B}\left(\nu_{n s}\right) .
$$

We see that in contrast to (5.4), in (5.12), $W_{\mathrm{CB}}$ is explicitly used. While for the short range interaction, (5.4) and (5.12) are equivalent, they treat long range interaction differently.

The associated Euler-Lagrange equations are

$$
\begin{aligned}
-\varepsilon^{2} \Delta \nu_{n s}+\frac{5}{3} \varepsilon^{2} \nu_{n s}^{7 / 3}-\left(\phi_{n s}+\phi_{s}\right) \nu_{n s}+\lambda \nu_{n s} & =0 \\
-\Delta \phi_{n s} & =4 \pi \varepsilon\left(m-\nu_{n s}^{2}\right) 1_{y\left(\Omega_{n s}\right)}
\end{aligned}
$$

where $\phi_{s}$ is the Coulombic potential generated by charges in the smooth region (taking the Cauchy-Born approximation).

Again, to calculate the force, we rewrite (5.12) in Lagrangian coordinates:

$$
\begin{aligned}
I_{\varepsilon}^{B}= & \int_{\Omega_{s}} W_{\mathrm{CB}}(\nabla u(x)) \mathrm{d} x \\
& +\varepsilon^{2} \int_{\Omega_{n s}} \nu_{n s}^{10 / 3} \operatorname{det}(I+A) \mathrm{d} x+\varepsilon^{2} \int_{\Omega_{n s}}\left|\nabla \nu_{n s}\right|^{2} \operatorname{det}(I+A) \mathrm{d} x \\
& +\frac{\varepsilon}{2} \iint_{\Omega_{n s} \times \Omega_{n s}} \frac{\left(\nu_{n s}^{2}-m\right)(y)\left(\nu_{n s}^{2}-m\right)\left(y^{\prime}\right)}{\left|y-y^{\prime}\right|} \operatorname{det}(I+A) \operatorname{det}\left(I+A^{\prime}\right) \mathrm{d} x \mathrm{~d} x^{\prime} \\
& +\varepsilon \iint_{\Omega_{s} \times \Omega_{n s}} \frac{\left(\nu_{s}^{2}-m\right)(y)\left(\nu_{n s}^{2}-m\right)\left(y^{\prime}\right)}{\left|y-y^{\prime}\right|} \operatorname{det}(I+A) \operatorname{det}\left(I+A^{\prime}\right) \mathrm{d} x \mathrm{~d} x^{\prime},
\end{aligned}
$$

where $y, y^{\prime}, A$, and $A^{\prime}$ are understood as $y(x), y\left(x^{\prime}\right), A(x)$, and $A\left(x^{\prime}\right)$ respectively. We compute the derivative (in the Gateaux sense) as:

$$
\frac{\delta}{\delta u} E_{\varepsilon}^{B}[h]=\int_{\Omega_{s}} \frac{\partial W_{\mathrm{CB}}}{\partial A_{i j}}(A(x)) \frac{\partial h_{i}}{\partial x_{j}} \mathrm{~d} x
$$




$$
\begin{aligned}
& +\varepsilon \iint_{\Omega_{s} \times \Omega_{n s}} \frac{\left(2 \nu_{s} \frac{\delta \nu_{s}}{\delta A_{i j}} \frac{\partial h_{i}}{\partial x_{j}}\right)(y)\left(\nu_{n s}^{2}-m\right)\left(y^{\prime}\right)}{\left|y-y^{\prime}\right|} \operatorname{det}(I+A) \operatorname{det}\left(I+A^{\prime}\right) \mathrm{d} x \mathrm{~d} x^{\prime} \\
& -\varepsilon \iint_{\Omega_{s} \times \Omega_{n s}} \frac{\left(\frac{\delta m}{\delta u}[h]\right)(y)\left(\nu_{n s}^{2}-m\right)\left(y^{\prime}\right)}{\left|y-y^{\prime}\right|} \operatorname{det}(I+A) \operatorname{det}\left(I+A^{\prime}\right) \mathrm{d} x \mathrm{~d} x^{\prime} \\
& +\varepsilon \iint_{\Omega_{s} \times \Omega_{n s}} \frac{\left(\nu_{s}^{2}-m\right)(y)\left(\nu_{n s}^{2}-m\right)\left(y^{\prime}\right)}{\left|y-y^{\prime}\right|} \frac{\partial \operatorname{det}(I+A)}{\partial A_{i j}} \frac{\partial h_{i}}{\partial x_{j}} \operatorname{det}\left(I+A^{\prime}\right) \mathrm{d} x \mathrm{~d} x^{\prime} \\
& -\varepsilon \iint_{\Omega_{s} \times \Omega_{n s}} \frac{\left(\nu_{s}^{2}-m\right)(y)\left(\nu_{n s}^{2}-m\right)\left(y^{\prime}\right)}{\left|y-y^{\prime}\right|^{3}}\left(y-y^{\prime}\right) \cdot h(x) \operatorname{det}(I+A) \operatorname{det}\left(I+A^{\prime}\right) \mathrm{d} x \mathrm{~d} x^{\prime} \\
& +\varepsilon^{2} \int_{\Omega_{n s}} \nu_{n s}^{10 / 3} \frac{\partial \operatorname{det}(I+A)}{\partial A_{i j}} \frac{\partial h_{i}}{\partial x_{j}} \mathrm{~d} x+\varepsilon^{2} \int_{\Omega_{n s}}\left|\nabla \nu_{n s}\right|^{2} \frac{\partial \operatorname{det}(I+A)}{\partial A_{i j}} \frac{\partial h_{i}}{\partial x_{j}} \mathrm{~d} x \\
& -\varepsilon \iint_{\Omega_{n s} \times \Omega} \frac{\left(\frac{\delta m}{\delta u}[h]\right)(y)\left(\nu^{2}-m\right)\left(y^{\prime}\right)}{\left|y-y^{\prime}\right|} \operatorname{det}(I+A) \operatorname{det}\left(I+A^{\prime}\right) \mathrm{d} x \mathrm{~d} x^{\prime} \\
& +\varepsilon \iint_{\Omega_{n s} \times \Omega} \frac{\left(\nu_{n s}^{2}-m\right)(y)\left(\nu^{2}-m\right)\left(y^{\prime}\right)}{\left|y-y^{\prime}\right|} \frac{\partial \operatorname{det}(I+A)}{\partial A_{i j}} \frac{\partial h_{i}}{\partial x_{j}} \operatorname{det}\left(I+A^{\prime}\right) \mathrm{d} x \mathrm{~d} x^{\prime} \\
& -\varepsilon \iint_{\Omega_{n s} \times \Omega} \frac{\left(\nu_{n s}^{2}-m\right)(y)\left(\nu^{2}-m\right)\left(y^{\prime}\right)}{\left|y-y^{\prime}\right|^{3}}\left(y-y^{\prime}\right) \cdot h(x) \operatorname{det}(I+A) \operatorname{det}\left(I+A^{\prime}\right) \mathrm{d} x \mathrm{~d} x^{\prime} .
\end{aligned}
$$

Comparing (5.17) with (5.9), we see that the interactions within the smooth regions are substituted by the derivative of the Cauchy-Born energy density. In some sense, model $\mathrm{B}$ fails to capture the long range interaction in the smooth region: since the Cauchy-Born approximation is used at the energy level, the energy functional only sees local terms in the smooth region.

For this reason, we expect that the model will generate "ghost force". To see this, we assume that the system is at equilibrium and $\Omega=\mathbb{R}^{3}$. By similar arguments as those for Model A, we get:

$$
\begin{aligned}
\frac{\delta}{\delta u} E_{\varepsilon}^{B}[h]= & \varepsilon \iint_{\Omega_{s} \times \Omega_{n s}} \frac{\left(2 \nu_{s} \frac{\delta \nu_{s}}{\delta A_{i j}} \frac{\partial h_{i}}{\partial x_{j}}\right)(x)\left(\nu_{n s}^{2}-m\right)\left(x^{\prime}\right)}{\left|x-x^{\prime}\right|} \mathrm{d} x \mathrm{~d} x^{\prime} \\
& -\varepsilon \iint_{\Omega_{s} \times \Omega_{n s}} \frac{\left(\frac{\partial m_{\mathrm{CB}}}{\partial A_{i j}} \frac{\partial h_{i}}{\partial x_{j}}\right)(x)\left(\nu_{n s}^{2}-m\right)\left(x^{\prime}\right)}{\left|x-x^{\prime}\right|} \mathrm{d} x \mathrm{~d} x^{\prime} \\
& +\varepsilon \iint_{\Omega_{s} \times \Omega_{n s}} \frac{\left(\nu_{s}^{2}-m\right)(x)\left(\nu_{n s}^{2}-m\right)\left(x^{\prime}\right)}{\left|x-x^{\prime}\right|} \frac{\partial \operatorname{det}(I+A)}{\partial A_{i j}} \frac{\partial h_{i}}{\partial x_{j}} \mathrm{~d} x \mathrm{~d} x^{\prime} \\
& -\varepsilon \iint_{\Omega_{s} \times \Omega_{n s}} \frac{\left(\nu_{s}^{2}-m\right)(x)\left(\nu_{n s}^{2}-m\right)\left(x^{\prime}\right)}{\left|x-x^{\prime}\right|^{3}}\left(x-x^{\prime}\right) \cdot h(x) \mathrm{d} x \mathrm{~d} x^{\prime} .
\end{aligned}
$$

However, now the integrands in (5.18) do not possess the symmetry property anymore. Hence in general they do not vanish, resulting in the generation of "ghost force" and the loss of uniform accuracy.

5.3. QM-continuum approximation of the KSDFT models. The QMcontinuum approximation for the KSDFT models can be developed in a similar fashion. Again, there are two different levels of Cauchy-Born approximation, resulting in two different QM-continuum models: Model A and Model B. Model A is free of "ghost forces" and in general, Model B generates "ghost forces". We will only describe these models, omitting other details. 
The wave functions associated with the smooth region are given by the CauchyBorn rule $\left\{\psi_{\mathrm{CB}, \alpha}\left(\frac{x-x_{i}}{\varepsilon} ; \nabla u\left(x_{i}\right)\right)\right\}$; the wave functions associated with the non-smooth region are $\left\{\psi_{n s, k}\right\}$. For the electron density, we define

$$
\begin{gathered}
\rho_{s}(y)=\rho_{\mathrm{CB}}\left(y ; \nabla u\left(x_{i}\right)\right)=\sum_{z_{j} \in L} \sum_{\alpha}\left|\psi_{\mathrm{CB}, \alpha}\left(\frac{x}{\varepsilon}-z_{j} ; \nabla u\left(x_{i}\right)\right)\right|^{2}, \\
\text { for } y \in(I+u)\left(\varepsilon \Gamma+x_{i}\right) ; \\
\rho_{n s}(y)=\sum_{k}\left|\psi_{n s, k}(y)\right|^{2}+\sum_{x_{i} \in \varepsilon L \cap \Omega_{s}} \sum_{\alpha}\left|\psi_{\mathrm{CB}, \alpha}\left(\frac{x-x_{i}}{\varepsilon} ; \nabla u\left(x_{i}\right)\right)\right|^{2} .
\end{gathered}
$$

Note that, in the smooth region, the electron density given by the Cauchy-Born rule is defined differently in each unit cell.

The energy functional for model $\mathrm{A}$ is then

$$
\begin{aligned}
I_{\varepsilon}^{A}= & \varepsilon^{2} \sum_{x_{i} \in \varepsilon L \cap \Omega_{s}} \sum_{\alpha} \int_{\mathbb{R}^{3}}\left|\nabla \psi_{\mathrm{CB}, \alpha}\left(\frac{x-x_{i}}{\varepsilon} ; \nabla u\left(x_{i}\right)\right)\right|^{2} \mathrm{~d} y \\
& +\int_{y\left(\Omega_{s}\right)} \epsilon_{\mathrm{xc}}\left(\rho_{s}\right) \rho_{s} \mathrm{~d} y+\frac{\varepsilon}{2} \iint_{y\left(\Omega_{s}\right) \times y\left(\Omega_{s}\right)} \frac{\left(\rho_{s}-m\right)(y)\left(\rho_{s}-m\right)\left(y^{\prime}\right)}{\left|y-y^{\prime}\right|} \mathrm{d} y \mathrm{~d} y^{\prime} \\
& +\varepsilon^{2} \sum_{k} \int_{\mathbb{R}^{3}}\left|\nabla \psi_{n s, k}\right|^{2} \mathrm{~d} y+\int_{y\left(\Omega_{n s}\right)} \epsilon_{\mathrm{xc}}\left(\rho_{n s}\right) \rho_{n s} \mathrm{~d} y \\
& +\frac{\varepsilon}{2} \iint_{y\left(\Omega_{n s}\right) \times y\left(\Omega_{n s}\right)} \frac{\left(\rho_{n s}-m\right)(y)\left(\rho_{n s}-m\right)\left(y^{\prime}\right)}{\left|y-y^{\prime}\right|} \mathrm{d} y \mathrm{~d} y^{\prime} \\
& +\varepsilon \iint_{y\left(\Omega_{s}\right) \times y\left(\Omega_{n s}\right)} \frac{\left(\rho_{s}-m\right)(y)\left(\rho_{n s}-m\right)\left(y^{\prime}\right)}{\left|y-y^{\prime}\right|} \mathrm{d} y \mathrm{~d} y^{\prime} .
\end{aligned}
$$

Similarly, we have for Model B:

$$
\begin{aligned}
I_{\varepsilon}^{B}= & \int_{\Omega_{s}} W_{\mathrm{CB}}(\nabla u(x)) \mathrm{d} x \\
& +\varepsilon^{2} \sum_{k} \int_{\mathbb{R}^{3}}\left|\nabla \psi_{n s, k}\right|^{2} \mathrm{~d} y+\int_{y\left(\Omega_{n s}\right)} \epsilon_{\mathrm{xc}}\left(\rho_{n s}\right) \rho_{n s} \mathrm{~d} y \\
& +\frac{\varepsilon}{2} \iint_{y\left(\Omega_{n s}\right) \times y\left(\Omega_{n s}\right)} \frac{\left(\rho_{n s}-m\right)(y)\left(\rho_{n s}-m\right)\left(y^{\prime}\right)}{\left|y-y^{\prime}\right|} \mathrm{d} y \mathrm{~d} y^{\prime} \\
& +\varepsilon \iint_{y\left(\Omega_{s}\right) \times y\left(\Omega_{n s}\right)} \frac{\left(\rho_{s}-m\right)(y)\left(\rho_{n s}-m\right)\left(y^{\prime}\right)}{\left|y-y^{\prime}\right|} \mathrm{d} y \mathrm{~d} y^{\prime} .
\end{aligned}
$$

\section{Dynamic QM-continuum model}

In this section, we study the dynamics of the system under QM-continuum approximation. We start with a new formulation for the smooth region in the QMcontinuum model based on the idea of fiber bundle dynamics [11]. We then consider the dynamics of the non-smooth region, which can be regarded as a generalization of the Car-Parrinello method [3]. We conclude with the discussion of dynamics for the whole system.

6.1. Fiber bundle structure of the smooth region. The smooth region in the QM-continuum model is most naturally described using a fiber bundle structure. 
Let us first recall the fiber bundle structure for such a multi-scale problem [11]: The macro-scale problem is the elastodynamics formulated in terms of the deformation gradient $A$ on the domain $\Omega$; the force field in the elastodynamics is given by the micro-structural variables that live on the fibers. Using the terminology of fiber bundle dynamics [11], we associate each point $x \in \Omega$ with a parametrization fiber $\gamma_{x}=\mathbb{R}^{3 n / 2}$. The state base manifold is $\mathbb{R}^{3 \times 3}=\left\{A, A \in \mathbb{R}^{3 \times 3}\right\}$, the space of deformation gradients. The micro-state fibers $\Gamma_{A}=\left\{\psi_{1}(\cdot ; A), \ldots, \psi_{n / 2}(\cdot ; A)\right\}$, where $\left\{\psi_{\alpha}\right\}_{\alpha=1}^{n / 2}$ are the wave functions.

The fiber bundle dynamics is given by

$$
\left\{\begin{aligned}
\rho_{0} \partial_{t}^{2} A & =\nabla(\nabla \cdot F(A)) \\
\frac{\delta}{\delta \psi_{\alpha}^{*}} W(A,\{\psi(\cdot ; A)\}) & =\sum_{\alpha^{\prime}, z_{j} \in L} \lambda_{\alpha \alpha^{\prime}, z_{j}} \psi_{\alpha^{\prime}}\left(z-z_{j} ; A\right) \\
\int \psi_{\alpha}^{*}\left(z-z_{i} ; A\right) \psi_{\alpha^{\prime}}\left(z-z_{j} ; A\right) \mathrm{d} z & =\delta_{\alpha \alpha^{\prime}} \delta_{i j} / \operatorname{det}(I+A) \\
F(A) & =\nabla_{A} W(A,\{\psi\})
\end{aligned}\right.
$$

Here $\rho_{0}$ is the density of the solid, $W(A,\{\psi\})$ is given by (4.30) and the $\lambda$ 's are Lagrangian multipliers for the normality constraints. The micro-structural electronic structure problem gives the model inputs $F(A)$ to the macro-scale model. Notice that the micro-dynamics on the fiber bundle is no longer the micro-scale dynamics for the original problem, in particular, we have rescaled the lattice constant from $\varepsilon$ to 1 .

The set of equations (6.1) corresponds to the Born-Oppenheimer adiabatic approximation, since $\{\psi\}$ is taken to minimize $W(A,\{\psi\})$ and so is assumed to be the ground state given by the displacement field. In the spirit of the Car-Parrinello method [3], we introduce a Car-Parrinello type of dynamics for the micro-structure fibers:

$$
\left\{\begin{aligned}
\rho_{0} \partial_{t}^{2} A= & \nabla(\nabla \cdot F(A)) \\
\mu \partial_{t}^{2} \psi_{\alpha}(\cdot ; A)+\eta \partial_{t} \psi_{\alpha}(\cdot ; A)= & \frac{\delta}{\delta \psi_{\alpha}^{*}} W(A,\{\psi\}) \\
& +\sum_{\alpha^{\prime}, z_{j} \in L} \lambda_{\alpha \alpha^{\prime}, z_{j}} \psi_{\alpha^{\prime}}\left(z-z_{j} ; A\right), \\
\int \psi_{\alpha}^{*}\left(z-z_{i} ; A\right) \psi_{\alpha^{\prime}}\left(z-z_{j} ; A\right) \mathrm{d} z= & \delta_{\alpha \alpha^{\prime}} \delta_{i j} / \operatorname{det}(I+A) \\
F(A)= & \partial_{A} W(A,\{\psi\})-\sum_{\alpha} \lambda_{\alpha \alpha, 0}(I+A)^{-1} / \operatorname{det}(I+A) .
\end{aligned}\right.
$$

Here $\mu$ is the artificial mass for the electrons and $\eta$ is a relaxation parameter. The expression for $F(A)$ is derived from a generalized Hellman-Feynman theorem [11], and the second term comes from the dependence of the orthonormality constraints on the deformation gradient $A$.

6.2. Dynamics in the non-smooth region. The dynamics for the nonsmooth region can be given by a Car-Parrinello type of dynamics. The QM-continuum energy functional (5.21) or (5.22) is used to derive the dynamics for the non-smooth region. For the non-smooth region, we keep explicitly the atomic positions $y_{j}$. The energy for the non-smooth region in the QM-continuum model, following (5.21) and 
(5.22), is given by

$$
\begin{aligned}
E_{n s}\left(\left\{y_{j}\right\},\left\{\psi_{n s}\right\}\right)= & \varepsilon^{2} \sum_{k} \int_{\mathbb{R}^{3}}\left|\nabla \psi_{n s, k}\right|^{2} \mathrm{~d} y+\int_{y\left(\Omega_{n s}\right)} \epsilon_{\mathrm{xc}}\left(\rho_{n s}\right) \rho_{n s} \mathrm{~d} y \\
& +\frac{\varepsilon}{2} \iint_{y\left(\Omega_{n s}\right) \times y\left(\Omega_{n s}\right)} \frac{\left(\rho_{n s}-m\right)(y)\left(\rho_{n s}-m\right)\left(y^{\prime}\right)}{\left|y-y^{\prime}\right|} \mathrm{d} y \mathrm{~d} y^{\prime} \\
& +\varepsilon \iint_{y\left(\Omega_{s}\right) \times y\left(\Omega_{n s}\right)} \frac{\left(\rho_{s}-m\right)(y)\left(\rho_{n s}-m\right)\left(y^{\prime}\right)}{\left|y-y^{\prime}\right|} \mathrm{d} y \mathrm{~d} y^{\prime} .
\end{aligned}
$$

Here $\rho_{s}$ and $\rho_{n s}$ are defined as in (5.19) and (5.20). The energy depends on the displacement field $u$ in the smooth region and also the wave functions in the smooth region given by the Cauchy-Born rule.

Given the energy, the extended Lagrangian for the non-smooth region is

$$
\mathcal{L}_{n s}=\sum_{j} M_{j}\left|\dot{y}_{j}\right|^{2}+\sum_{k} \int_{\mathbb{R}^{3}} \mu\left|\dot{\psi}_{n s, k}\right|^{2} \mathrm{~d} y-E_{n s}\left(\left\{y_{j}\right\},\left\{\psi_{n s}\right\}\right) .
$$

Here $M_{j}$ are the masses for the atoms and $m$ is the artificial mass for the electrons as in the original Car-Parrinello method. The extended Lagrangian is the same with the original Car-Parrinello molecular dynamics, except that $E_{n s}$ is given by the QMcontinuum model. From the extended Lagrangian, it is standard to write down the dynamics for the atomic positions and wave functions

$$
\begin{aligned}
M_{j} \partial_{t}^{2} u_{j} & =-\frac{\delta E_{n s}\left(\left\{u_{j}\right\},\left\{\psi_{n s}\right\}\right)}{\delta u_{j}} \\
\mu \partial_{t}^{2} \psi_{n s, k}= & -\frac{\delta E_{n s}\left(\left\{u_{j}\right\},\left\{\psi_{n s}\right\}\right)}{\delta \psi_{n s, k}^{*}} \\
& +\sum_{k^{\prime}} \Lambda_{k, k^{\prime}}^{n s} \psi_{n s, k^{\prime}}+\sum_{\alpha, x_{i} \in \varepsilon L \cap \Omega_{s}} \Lambda_{k, \alpha, x_{i}}^{s} \psi_{s, \alpha, x_{i}} .
\end{aligned}
$$

Here the $\Lambda$ 's are Lagrangian multipliers for the orthonormality conditions and $\psi_{s, \alpha, x_{i}}$ is the $\alpha$-th wave function localized at $(I+u)\left(x_{i}\right)$ in the smooth region. The derivative of $E_{n s}$ with respect to $\psi_{n s}$ can be calculated explicitly as

$$
-\frac{\delta E_{n s}\left(\left\{u_{j}\right\},\left\{\psi_{n s}\right\}\right)}{\delta \psi_{n s, k}^{*}}=\varepsilon^{2} \Delta \psi_{n s, k}-V_{\mathrm{xc}}\left(\rho_{n s}\right) \psi_{n s, k}+\phi(y) \psi_{n s, k} .
$$

6.3. Dynamics of the whole system. For the dynamics of the whole system with the QM-continuum model, we couple together the fiber bundle dynamics for the smooth region and the generalized Car-Parrinello dynamics for the non-smooth region. We make several remarks about the coupling.

Recall that for the smooth region, we use the continuum displacement field $u$, while for the non-smooth region, we keep explicitly the atomic positions $y_{j}$. Therefore, we need to determine the boundary condition of $u$ at the interface between smooth and non-smooth regions. The boundary condition can be given by the locations of atoms in the non-smooth region through an operator which we denote as $\mathcal{P}$ :

$$
u_{s}(x)=\mathcal{P}\left(\left\{u_{j}\right\}\right), \quad x \text { on } \partial \Omega_{n s} .
$$

The reconstruction operator $\mathcal{P}$ is in general an interpolation operator. 
Another source of coupling between the smooth and non-smooth regions is that, in the non-smooth energy (6.3), we need the wave functions $\left\{\psi_{s}\right\}$ from the fiber bundle dynamics in the smooth region. Hence, we need to construct them on the physical space through the corresponding micro-state variables on the fibers. More specifically, what we have are $\psi_{\alpha}\left(z ; A\left(x_{i}\right)\right)$ on the fiber over $A\left(x_{i}\right)$; however, we need on the physical space $\psi_{s, \alpha, x_{i}}(y)$, which are the $\alpha$-th wave functions localized at $(I+u)\left(x_{i}\right)$, with variable $y$ on the physical space (not the fiber). One natural way to do the reconstruction is to set

$$
\psi_{s, \alpha, x_{i}}(y)=\psi_{\alpha}\left(\frac{x(y)-x_{i}}{\varepsilon} ; A\left(x_{i}\right)\right) ;
$$

that is, we rescale $\psi_{\alpha}$ and patch them together on the physical space to give the wave functions in the smooth region. Of course, in practice, the elastic dynamics in the smooth region is discretized. We do not have the information of $\psi_{\alpha}$ on fiber $\gamma_{x}$ for every $x \in \Omega$, since the grid size is generally much larger than the lattice constant $\varepsilon$. However, the $\psi_{s, \alpha, x_{i}}(y)$ can still be reconstructed using interpolation.

The dynamics in the smooth region is as described in Section 6.1. We impose the boundary condition of $u(6.8)$ at the interface between the smooth and non-smooth regions. The dynamics in the non-smooth region is as described in Section 6.2 with wave functions in the smooth region reconstructed through the information on the micro-structure fibers. The coupled dynamics can be used to study the dynamics of the system under the QM-continuum approximation.

\section{Conclusion}

We discussed the continuum limit of DFT models for crystalline solids. The treatment here is formal. However, it provides much important information for understanding these models and for analyzing the coupling of the original models with models coming from continuum theory. Through the analysis of two different levels of QM-continuum approximation of these models, we conclude that Model A is more accurate since it does not induce "ghost forces" at the interface between the smooth and the non-smooth regions, in contrast to Model B.

We also introduced a new formulation for the dynamical QM-continuum approximation based on the fiber bundle structure for the smooth region. This allows us to extend naturally the Car-Parrinello method to the QM-continuum setting. The new formulation also provides an alternative perspective for the QM-continuum model which is useful in numerical computations.

Finally, since the arguments in this paper are quite formal, let us comment briefly on their validity and more generally the usefulness of these results. Concerning the TFW model, since there is already a well-established mathematical theory, we believe it should be possible, although quite tedious, to establish the validity of the asymptotic analysis presented here. Indeed, the results of Blanc et al. [1] can already be viewed as a partial justification of the leading order asymptotics. Higher order results are also useful, particularly for numerical purposes [14]. For KSDFT, however, mathematical results are rather sparse. In particular, as was observed by one of the referees, the KSDFT functional is non-convex; therefore we do not expect uniqueness for the solutions. Nevertheless, this does not preclude the possibility of establishing the validity of the asymptotic results using local arguments, along the lines in [13]. This is indeed one strategy that we are pursuing.

Acknowledgment. We are grateful to Carlos García-Cervera and Jingfang 
Huang for very helpful discussions. This work is partially supported by ONR grant

N00014-01-1-0674, DOE grant FG02-03ER25587 and NSF grant DMS-0407866.

\section{REFERENCES}

[1] X. Blanc, C. Le Bris and P.L. Lions, From molecular models to continuum mechanics, Arch. Ration. Mech. Anal., 164, 341-381, 2002.

[2] J.Q. Broughton, F.F. Abraham, N. Bernstein and E. Kaxiras, Concurrent coupling of length scales: methodology and application, Phys. Rev. B, 60, 2391-2402, 1999.

[3] R. Car and M. Parrinello, Unified approach for molecular dynamics and density-functional theory, Phys. Rev. Lett., 55, 2471-2474, 1985.

[4] I. Catto, C. Le Bris and P.L. Lions, The Mathematical Theory of Thermodynamic Limits: Thomas-Fermi Type Models, Clarendon Press, Oxford, 1998.

[5] I. Catto, C. Le Bris and P.L. Lions, On the thermodynamic limit for Hartree-Fock type models, Ann. Inst. Henri Poincaré, 18, 687-760, 2001.

[6] I. Catto, C. Le Bris and P.L. Lions, On some periodic Hartree-type models for crystals, Ann. Inst. Henri Poincaré, 19, 143-190, 2002.

[7] N. Choly, G. Lu, W. E and E. Kaxiras, Multiscale simulations in simple metals: a densityfunctional-based methodology, Phys. Rev. B, 71, 094-101, 2005.

[8] W.A. Curtin and R.E. Miller, Atomistic/continuum coupling in computational materials science, Modelling Simul. Mater. Sci. Eng., 11, R33-R68, 2003.

[9] W. E and Z. Huang, A dynamic atomistic-continuum method for the simulation of crystalline materials, J. Comp. Phys., 182, 234-261, 2002.

[10] W. E and J. Lu, The elastic continuum limit of the tight binding model, Chinese Ann. Math. Ser. B, in press.

[11] W. E and J. Lu, Seamless multiscale modeling via dynamics on fiber bundles, Commun. Math. Sci., 5, 649-663, 2007.

[12] W. E, J. Lu and J. Yang, Uniform accuracy of the quasicontinuum method, Phys. Rev. B, 74, 214115, 2006.

[13] W. E and P.B. Ming, Cauchy-Born rule and the stability of crystalline solids: static problems, Arch. Ration. Mech. Anal., 183, 241-297, 2007.

[14] C.J. García-Cervera, An efficient real space method for orbital-free density-functional theory, Commun. Comput. Phys., 2, 334-357, 2007.

[15] V. Gavini, K. Bhattacharya and M. Ortiz, Quasi-continuum orbital-free density-functional theory: aroute to multi-million atom non-periodic DFT calculation, J. Mech. Phys. Solids, 55, 697-718, 2007.

[16] V. Gavini, J. Knap, K. Bhattacharya and M. Ortiz, Non-periodic finite-element formulation of orbital-free density functional theory, J. Mech. Phys. Solids, 55, 669-696, 2007.

[17] E.H. Lieb and B. Simon, The Thomas-Fermi theory of atoms, molecules and solids, Adv. Math., 23, 22-116, 1977.

[18] R. Martin, Electronic Structure: Basic Theory And Practical Methods, Cambridge Univ. Press, Cambridge, 2004.

[19] D. Negrut, M. Anitescu, A. El-Azab and P. Zapol, Quasicontinuum-like reduction of DFT calculations of nanostructures, preprint.

[20] R.G. Parr and W. Yang, Density-Functional Theory of Atoms And Molecules, Oxford Univ. Press, New York, 1989.

[21] T. Shimokawa, J.J. Mortensen, Schiotz. J and K.W. Jacobsen, Matching conditions in the quasicontinuum method: removal of the error introduced at the interface between the coarsegrained and fully atomistic region, Phys. Rev. B, 69, 214104, 2004.

[22] E.B. Tadmor, M. Ortiz and R. Phillips, Quasicontinuum analysis of defects in solids, Philos. Mag. A, 73, 1529-1963, 1996.

[23] G.H. Wannier, The structure of electronic excitation levels in insulating crystals, Phys. Rev., 52, 191-197, 1937. 